OUTP 94-14 P

HD-THEP-94-15

hep-th/9408132

August 1994

\title{
COLEMAN-WEINBERG PHASE TRANSITION IN TWO-SCALAR MODELS
}

\author{
S. Bornholdtt ${ }^{\mathrm{a}}$ 円, N. Tetradis ${ }^{\mathrm{b}}$ and C. Wetterich ${ }^{\mathrm{a}}$ \\ a)Institut für Theoretische Physik, Universität Heidelberg, \\ Philosophenweg 16, 69120 Heidelberg, Germany \\ b)Theoretical Physics, University of Oxford, \\ 1 Keble Road, Oxford OX1 3NP, U.K.
}

\begin{abstract}
We explore the Coleman-Weinberg phase transition in regions outside the validity of perturbation theory. For this purpose we study a Euclidean field theory with two scalars and discrete symmetry in four dimensions. The phase diagram is established by a numerical solution of a suitable truncation of exact non-perturbative flow equations. We find regions in parameter space where the phase transition (in dependence on the mass term) is of the second or the first order, separated by a triple point. Our quantitative results for the first order phase transition compare well to the standard perturbative Coleman-Weinberg calculation of the effective potential.
\end{abstract}

\footnotetext{
${ }^{1}$ Address after August $1^{\text {st }}$ : Institut für Theoretische Physik, Universität Kiel, Olshausenstr. 6, 24118 Kiel, Germany.
} 
During the last two decades, the perturbative Coleman-Weinberg approach [1] to the calculation of the effective potential by a loop expansion has been widely used in studying field theories that exhibit spontaneous symmetry breaking. Within the standard model and for low masses of the Higgs boson this approach predicts a weakly first order phase transition in dependence on the mass parameter. This feature has been used to derive a lower bound on the mass of the Higgs boson [2]. A generalization of the Coleman-Weinberg calculation for non-vanishing temperature suggests also a first order phase transition in dependence on the temperature, at least if the mass of the Higgs Boson is sufficiently small [3]. The observation that the baryon asymmetry may have been created during the electroweak phase transition in the very early universe [4] considerably renewed the interest in this subject [5]. Indeed, this hypothesis received much encouragement from the fact that the loop expansion for the potential predicts a first order phase transition with periods outside thermodynamic equilibrium, which is one of the three main ingredients for the generation of net baryon number [6]. When the experimental bounds for the Higgs mass [7] began to diverge from the theoretical expectations [8], two-Higgs-doublet extensions of the standard model entered the scene. They provide additional CP violation (which is another requirement for baryogenesis). Also perturbative calculations of the effective potential suggest that the phase transition in these models can be sufficiently strongly first order and nevertheless remain compatible with the experimental mass bound [9]. However, it is not clear whether these perturbative methods give the right picture of the phase transition, since the same methods fail to give a correct description of the second order high temperature phase transition [12] in models with only one scalar. A complete description of the phase transitions in two-scalar models is still lacking.

Another wide range of application of effective potentials concerns inflationary cosmology. In the model proposed originally by Guth [10] as well as in later models [11], an effective potential of the Coleman-Weinberg type has been used to allow for a phase of exponential expansion of the early universe. These models often do not specify what the underlying theory should be, but they nevertheless assume that a general field theoretical framework exists which produces this type of potential.

The high temperature phase transition can be understood in terms of the temperature dependent effective potential $U(\varphi, T)$ for the scalar field $\varphi$. The crucial feature of a first order transition is the existence of two local minima at $\varphi=0$ and $\varphi \neq 0$, separated by a barrier, in a certain temperature range. Similarly, if the zero temperature theory exhibits this feature for a certain range of the mass parameter one speaks about a first order transition in dependence on the mass parameter. By continuity in $T$ for $T=0$, the two phenomena are closely related. The renormalization group improved loop expansion is believed to work well near the absolute minimum of the effective potential. Already in the original paper [1], however, it was pointed out that the description of possible additional relative minima is not within the range of applicability of perturbation theory. In the phase with spontaneous symmetry breaking the validity of the one-loop results for the form of the effective potential at the origin $(\varphi=0)$ has not been established so far. However, a reliable description of both the absolute and the relative minimum is crucial 
for a firm establishment of a first order phase transition.

In this letter we perform a non-perturbative analysis which is capable of dealing with the above problem. It confirms the picture arising from the results of the ColemanWeinberg loop expansion for the effective potential in the vicinity of the phase transition. We employ a new method based on the effective average action [13] which is the analogue of the block spin action [14 for continuous space. The dependence of the effective average action $\Gamma_{k}$ on the average scale $k$ (one averages over a volume $\sim k^{-d}$ ) is given by an exact evolution equation 15 If. In the limit $k \rightarrow 0, \Gamma_{k}$ becomes the usual effective action (the generating functional of the 1PI Greens functions). For $k>0$ the effective average potential $U_{k}$ (the non-derivative part of $\Gamma_{k}$ ) is not necessarily convex. This permits the study of several minima of different depth. The effective average potential becomes convex in the limit $k \rightarrow 0$ [17.

We want to solve the flow equation which describes how the effective average potential changes its shape as $k$ decreases from some high scale (UV cutoff) to zero. For this purpose we first employ a truncation of the exact non-perturbative evolution equation by neglecting wave function renormalization effects and $\varphi^{6}$ and higher effective vertices. Even in this approximation our approach goes beyond the usual gap equation for the mass term since it effectively contains a similar self-coupled equation for the quartic scalar coupling. It also properly accounts for mass effects in the scale dependence of 1PI vertices and describes how particles with mass $m$ much larger than $k$ decouple from the running of the couplings with varying $k$. These features are not included in the ColemanWeinberg one-loop calculation. The standard perturbative result for the $\beta$-functions of the quartic scalar couplings is recovered from our results for small couplings and vanishing masses. In a second step we improve the truncation by including the effects of $\varphi^{6}$ and $\varphi^{8}$ couplings. This permits the investigation of potentials with a rich variety of shapes. For example, the potential at large $k$ may have only one minimum (up to the degeneracy due to symmetry), whereas at lower $k$ new relative minima may appear as a result of integrating out quantum modes with momenta larger than $k$. Following the evolution of the different extrema permits the determination of the nature of the phase transition. The reliability of our non-perturbative method for second order phase transitions has recently been demonstrated by computing the high temperature phase transition of the $\mathrm{O}(\mathrm{N})$ symmetric scalar theory and the corresponding critical exponents to a high precision [12]. Here we address a model with a first order phase transition.

We consider a simple scalar field theory with two scalar degrees of freedom in four dimensions. We limit ourselves to the zero temperature case and study the phase transition with varying mass parameter. After discussing the tree level potential we first recall the results of the Coleman-Weinberg loop expansion. Then the method of the effective average action is briefly introduced and the formalism for the model under investigation is given. We study spontaneous symmetry breaking in a model of two scalar fields $\varphi_{1}, \varphi_{2}$

\footnotetext{
${ }^{2}$ See ref. [16] for other version of exact renormalization group equations.
} 
with a classical potential symmetric under $\varphi_{1} \rightarrow-\varphi_{1}, \varphi_{2} \rightarrow-\varphi_{2}, \varphi_{1} \rightarrow \varphi_{2}$

$$
V=-\mu^{2}\left(\rho_{1}+\rho_{2}\right)+\frac{\lambda}{2}\left(\rho_{1}^{2}+\rho_{2}^{2}\right)+g \rho_{1} \rho_{2}
$$

where $\rho_{1}=\frac{1}{2} \varphi_{1}^{2}$ and $\rho_{2}=\frac{1}{2} \varphi_{2}^{2}$. This potential is bounded from below provided $g>$ $-\lambda, \lambda>0$. It is also convenient to use the parametrization

$$
V=-\mu^{2}\left(\rho_{1}+\rho_{2}\right)+\lambda\left[\frac{1}{2}\left(\rho_{1}+\rho_{2}\right)^{2}+x \rho_{1} \rho_{2}\right]
$$

with $x=(g-\lambda) / \lambda$ measuring the deviation from the case with $O(2)$ symmetry discussed earlier with the method of the effective average action [13, 12]. The structure of this potential is described by three different phases. For $\mu^{2}<0$ the classical theory is in the symmetric phase with the minimum lying at the origin (S phase). If $\mu^{2}>0$, one or both of the scalar fields develop a vacuum expectation value. One finds the minimum on the axes for $x>0$ (AX phase) or between the axes for $x<0$ (M phase). For $x=0$ the potential exhibits an additional $\mathrm{O}(2)$ symmetry. Due to the symmetries of the potential, the minima on the axes occur symmetrically at $\left(\rho_{1}=\mu^{2} / \lambda, \rho_{2}=0\right)$ and $\left(\rho_{1}=0, \rho_{2}=\mu^{2} / \lambda\right)$. In the $\mathrm{M}$ phase, the minimum is always located between the axes at $\rho_{1}=\rho_{2}=\mu^{2} /(g+\lambda)$.

The renormalization group improved one-loop potential can be parametrized in terms of scale dependent couplings $\lambda$ and $g$. An important ingredient is the coupling $g$ which couples the two scalar fields. In a study of the scale dependence of $\lambda$ this coupling appears in the renormalization group equation for $\lambda$ (and vice versa). Two interesting scenarios might be induced, which seem to indicate first order phase transitions. First, a term $\sim g^{2}$ appears in the $\beta$-function for $\lambda$, so that $\lambda$, instead of running to zero for $k \rightarrow 0$ as in the $\mathrm{O}(\mathrm{N})$ symmetric $\varphi^{4}$-theory, may reach zero during a finite renormalization interval and then be driven to negative values. For $\mu^{2}>0$ (AX phase) this would correspond to turning the minimum of the effective potential into a saddlepoint. This situation is very similar to the scale dependence of the quartic scalar coupling in the Abelian Higgs model originally studied by Coleman and Weinberg [1] (with $g$ playing the role of $e^{2}$ ) and is generally believed to indicate a first order phase transition. A second interesting case occurs for negative couplings $g$. When $g$ becomes smaller than $-\lambda$, again the minimum flips over. Let us see more quantitatively what standard perturbation theory tells us. The one-loop renormalization group equation for the quartic coupling $\lambda$ reads

$$
\frac{d \lambda}{d t}=\frac{1}{16 \pi^{2}}\left(9 \lambda^{2}+g^{2}\right)
$$

with a logarithmic renormalization scale $t=\ln k$. Indeed, If $g^{2}$ is large enough, $\lambda$ runs to zero within a finite interval of $t$. Since the evolution of the mixed coupling $g$ turns out to be

$$
\frac{d g}{d t}=\frac{1}{16 \pi^{2}}\left(6 \lambda g+4 g^{2}\right)
$$

this interesting feature always shows up for a sufficiently large starting value $g_{0} / \lambda_{0}$. In the case of negative couplings $g$ we are interested in what happens for $x$ approaching -2 
or $g \simeq-\lambda$. Near $x \simeq-2$ one finds

$$
\frac{d x}{d t} \simeq \frac{\lambda}{2 \pi^{2}}
$$

which, for large enough $\lambda$ and initial value of $x$ close to -2 , leads to values of $x<-2$ thus flipping the minimum into a saddlepoint! In the same region one has

$$
\frac{d \lambda}{d t} \simeq \frac{5 \lambda^{2}}{8 \pi^{2}}
$$

ensuring that there is always a natural parameter range where this phenomenon occurs, indicating again a first order phase transition.

However, some questions remain open. The above renormalization group equations do not depend on the masses of the theory and are in this respect "scale invariant". This is a good approximation for mass scales much larger than the characteristic masses of the theory. For a first order phase transition the masses do not vanish even for the critical parameters. For scales below the critical mass the running of the dimensionless couplings should stop. For a precise understanding of the character of the phase transition we want to take these "threshold effects" into account. We therefore introduce a scale $k$ which acts as an infrared cutoff for the quantum fluctuations, independently of all other possible infrared cutoffs such as field dependent masses and external momenta. This replaces the effective potential $U$ by an "effective average potential" $U_{k}$ in which only field modes with momenta larger than $k$ are integrated over.

We start with the evolution equation 15 describing the dependence of the effective average potential $U_{k}$ on the average scale $k(t=\ln k)$ in arbitrary dimensions $d$

$$
\frac{\partial U_{k}}{\partial t}\left(\rho_{1}, \rho_{2}\right)=v_{d} \int_{0}^{\infty} d x x^{\frac{d}{2}-1} \frac{\partial P}{\partial t}\left\{\frac{1}{P+M_{1}^{2}}+\frac{1}{P+M_{2}^{2}}\right\}
$$

Here the variable $x$ denotes momentum squared and $v_{d}^{-1}=2^{d+1} \pi^{\frac{d}{2}} \Gamma\left(\frac{d}{2}\right)$. The inverse average propagator

$$
P=\frac{x}{1-\exp \left(-\frac{x}{k^{2}}\right)}
$$

contains an effective infrared cutoff for the modes with $x<k^{2}$. The eigenvalues of the mass matrix

$$
M_{1,2}^{2}=\frac{1}{2}\left(U_{1}+U_{2}\right)+U_{11} \rho_{1}+U_{22} \rho_{2} \pm \sqrt{\left[\frac{1}{2}\left(U_{1}-U_{2}\right)+U_{11} \rho_{1}-U_{22} \rho_{2}\right]^{2}+4 U_{12}^{2} \rho_{1} \rho_{2}}
$$

are determined by the partial derivatives of the effective average potential $U_{1} \equiv \partial U_{k} / \partial \rho_{1}$, $U_{12} \equiv \partial^{2} U_{k} / \partial \rho_{1} \partial \rho_{2}$ etc. We note the close resemblance of the flow equation (7) to a renormalization group improved one-loop calculation with infrared cutoff (for $k=0$ one has $P=x$ ). Up to neglecting effects from the wave function renormalization this flow equation is an exact non-perturbative equation [15, 12]. The appearance of renormalized and $\rho$-dependent mass terms instead of bare mass terms turns eq. (7) into a self-coupled 
equation with a structure similar to the gap equation for the renormalized mass. A gap equation for the masses can indeed be obtained by computing $\partial M_{1,2}^{2} / \partial t$ using partial derivatives of eq. (77) with respect to $\rho_{1}$ and $\rho_{2}$. Similar self-coupled equations for the quartic couplings follow from taking second partial derivatives of eq. (7). After performing the momentum integration the evolution equation (7) becomes a partial differential equation for $U_{k}\left(\rho_{1}, \rho_{2}, t\right)$. This is difficult to solve explicitly and we will first discuss an approximate solution based on neglecting partial derivatives of higher than second order, i.e. terms $\sim U_{111}$ or $\varphi^{6}$ couplings and so on. This approximation is too crude to account well for complex structures of $U_{k}$, as for example several inequivalent local minima (see below). Nevertheless it is a valid approximation as long as $k$ is much larger than the physical masses. Before turning to less restrictive truncations we use the quartic potential here as an illustration of our method. The evolution of the potential is then described by a set of three renormalized parameters which depend on the average scale $k$. We choose $\lambda=U_{11}, x=U_{12} / U_{11}-1$ where partial derivatives are evaluated at the minimum, and a third parameter which is either a mass $m^{2}=U_{1}$ (S phase) or the distance $\rho_{0}$ of the minimum from the origin, i.e. $\rho_{0}=\left(\rho_{1}\right)_{\min }$ (AX phase) or $\rho_{0}=\left(\rho_{1}\right)_{\min }+\left(\rho_{2}\right)_{\min }(\mathrm{M}$ phase). At the ultraviolet cutoff $\Lambda$, the physics is described by the classical potential $V$, while at some lower scale $k<\Lambda$ by the effective average potential $U_{k}$ which is obtained from eq. (7) with the initial condition $U_{\Lambda}=V$. The effective average potential interpolates between the classical potential $(k=\Lambda)$ and the effective potential $(k=0)$ [15]. A solution of the evolution equation for $k \rightarrow 0$ then leads to the effective 1PI vertices at zero momentum. The effective average potential does not in general stay a quartic potential but receives higher order terms from the renormalization group flow. Near a unique minimum, however, it can be taken to a good approximation to be of the same form as in eq. (2) with renormalized parameters $\mu^{2}, \lambda$, and $x$. In this case our truncation of higher order couplings (like $\varphi^{6}$ terms) should be a valid approximation. The evolution of the renormalized parameters with changing scale $k$ is derived from eq. (7) by partial differentiation with respect to $\left.\rho_{1}, \rho_{2}\right]^{\text {? }}$.

We next present the evolution equations for the three relevant couplings for the $\mathrm{S}$ regime, where the partial derivatives defining the running parameters are evaluated at the minimum which lies at the origin $\left(\rho_{10}=\rho_{20}=0\right)$. This is always the case for $m^{2}=-\mu^{2}>0$. One obtains

$$
\begin{aligned}
\frac{d m^{2}}{d t} & =-2 v_{d} k^{d-2}(4+x) \lambda l_{1}^{d} s_{1}^{d}\left(\frac{m^{2}}{k^{2}}\right) \\
\frac{d \lambda}{d t} & =2 v_{d} k^{d-4}\left(10+2 x+x^{2}\right) \lambda^{2} l_{2}^{d} s_{2}^{d}\left(\frac{m^{2}}{k^{2}}\right)
\end{aligned}
$$

\footnotetext{
${ }^{3}$ Note that in the AX phase the evolution equations for $\lambda=U_{11}$ would differ from the one defined as $\lambda=U_{22}$ by higher order terms in powers of $\lambda$ and $x$. This is due to the choice of a particular truncation of the effective average potential $U_{k}$ which we fix by defining $\lambda$ always through partial derivatives in the direction of the minimum as viewed from the origin.
} 


$$
\frac{d x}{d t}=-2 v_{d} k^{d-4}(x+1) x(x-2) \lambda l_{2}^{d} s_{2}^{d}\left(\frac{m^{2}}{k^{2}}\right)
$$

where $l_{1}^{d}$ and $l_{2}^{d}$ are constants of order one defined by

$$
l_{n}^{d}=\frac{n}{2} k^{2 n-d} \int_{0}^{\infty} d x x^{\frac{d}{2}-1} \frac{\partial P}{\partial t} P^{-n-1} .
$$

The "threshold functions" $s_{n}^{d}\left(\frac{w}{k^{2}}\right)$ are given by

$$
s_{n}^{d}\left(\frac{w}{k^{2}}\right)=\frac{n}{2}\left(l_{n}^{d}\right)^{-1} k^{2 n-d} \int_{0}^{\infty} d x x^{\frac{d}{2}-1} \frac{\partial P}{\partial t}(P+w)^{-n-1}
$$

and depend on the dimensionless ratio $w / k^{2}$. They approach unity for vanishing argument, vanish for large arguments and describe the decoupling of particles with mass greater than $k$. More details can be found in refs. [13, 12]. Another useful equation, though redundant, is the running of the coupling $g$

$$
\frac{d g}{d t}=2 v_{d} k^{d-4}\left(10+14 x+4 x^{2}\right) \lambda^{2} l_{2}^{d} s_{2}^{d}\left(\frac{m^{2}}{k^{2}}\right)
$$

with $g=(1+x) \lambda$. We observe that for $d=4$ and $m^{2}=0$ we recover from eqs. (11) and (15) the standard one-loop $\beta$-functions of eqs. (3) and (4) (making use of $v_{4}=1 / 32 \pi^{2}$ and $l_{2}^{4}=1$ ).

The only non-perturbative content in the approximation of eqs. (11) and (15) arises through the threshold functions $s_{2}^{d}$ which describe in a natural way that particles with $m^{2} \gg k^{2}$ should not influence the variation of the couplings with an infrared scale $k$. The presence of mass thresholds in the $\beta$-functions, though physically very reasonable, is not seen in many versions of the renormalization group equations. In our approach it arises naturally from eq. (7), together with an additional equation (10) for the scale dependence of the mass term. The "quadratic renormalization" of the mass term given by eq. (10) incorporates in the renormalization group framework the physics related to the "quadratic divergences". Although known in practice since a long time in the Wilson approach to the renormalization group (for example in lattice studies) this equation is often missing in the framework of perturbative renormalization group equations. The reason is simply that if no mass scale other than $m^{2}$ is present in a given formulation of the renormalized potential, ratios like $m^{2} / k^{2}$ cannot be formed and such approaches are necessarily blind to mass thresholds.

In the $\mathrm{AX}$ regime $(x>0)$ we choose the minimum at $\left(\rho_{10}=\rho_{0}>0, \rho_{20}=0\right)$. Here $\rho_{0}$ is determined by $U_{1}\left(\rho_{0}, 0\right)=0$ and one has $U_{2}\left(\rho_{0}, 0\right)=(g-\lambda) \rho_{0}=x \lambda \rho_{0}$. The evolution equations now refer to the couplings defined at $\left(\rho_{0}, 0\right)$

$$
\frac{d \rho_{10}}{d t}=2 v_{d} k^{d-2} l_{1}^{d}\left[3 s_{1}^{d}(2 \lambda \kappa)+(1+x) s_{1}^{d}(x \lambda \kappa)\right]
$$




$$
\begin{aligned}
& \frac{d \lambda}{d t}=2 v_{d} k^{d-4} l_{2}^{d} \lambda^{2}\left[9 s_{2}^{d}(2 \lambda \kappa)+(1+x)^{2} s_{2}^{d}(x \lambda \kappa)\right] \\
& \frac{d x}{d t}=2 v_{d} k^{d-4} l_{2}^{d} \lambda \frac{x(1+x)}{1-\frac{x}{2}}\left[9 s_{2}^{d}(2 \lambda \kappa)+\left(\frac{x^{2}}{2}-2 x-7\right) s_{2}^{d}(x \lambda \kappa)\right] \\
& \frac{d g}{d t}=2 v_{d} k^{d-4} l_{2}^{d} \lambda^{2}\left[\frac{9+\frac{27}{2} x+\frac{9}{2} x^{2}}{1-\frac{x}{2}} s_{2}^{d}(2 \lambda \kappa)+(1+x) \frac{1-\frac{11}{2} x-2 x^{2}}{1-\frac{x}{2}} s_{2}^{d}(x \lambda \kappa)\right]
\end{aligned}
$$

with $\kappa=\rho_{0} / k^{2}$. If finally $-2<x<0$ and both scalars develop a vacuum expectation value (M regime) we use $\rho_{10}=\rho_{20}=\frac{1}{2} \rho_{0}$ with $\rho_{0}$ determined by $U_{1}\left(\frac{1}{2} \rho_{0}, \frac{1}{2} \rho_{0}\right)=$ $U_{2}\left(\frac{1}{2} \rho_{0}, \frac{1}{2} \rho_{0}\right)=0$. The mass eigenvalues are given by $M_{1}^{2}=(2+x) \lambda \rho_{0}, M_{2}^{2}=-x \lambda \rho_{0}$ and the evolution equations read

$$
\begin{aligned}
& \frac{d \rho_{0}}{d t}=2 v_{d} k^{d-2} l_{1}^{d}\left[3 s_{1}^{d}((2+x) \lambda \kappa)+\frac{2-x}{2+x} s_{1}^{d}(-x \lambda \kappa)\right] \\
& \frac{d \lambda}{d t}=2 v_{d} k^{d-4} l_{1}^{d} \frac{3 x \lambda}{\kappa} \frac{1+\frac{x}{4}}{1+x}\left[s_{1}^{d}((2+x) \lambda \kappa)-s_{1}^{d}(-x \lambda \kappa)\right] \\
&+2 v_{d} k^{d-4} l_{2}^{d} \lambda^{2}\left[9\left(1+\frac{x}{2}\right)^{2} s_{2}^{d}((2+x) \lambda \kappa)+\left(1-\frac{x}{2}\right)^{2} s_{2}^{d}(-x \lambda \kappa)\right] \\
& \frac{d x}{d t}=-2 v_{d} k^{d-4} l_{1}^{d} \frac{3}{\kappa} \frac{2+x}{1+x}\left(x+\frac{x^{2}}{4}\right)\left[s_{1}^{d}((2+x) \lambda \kappa)-s_{1}^{d}(-x \lambda \kappa)\right] \\
& \quad-2 v_{d} k^{d-4} l_{2}^{d} x \lambda\left[9\left(1+\frac{x}{2}\right)^{2} s_{2}^{d}((2+x) \lambda \kappa)+\left(1-\frac{x}{2}\right)^{2} s_{2}^{d}(-x \lambda \kappa)\right] \\
& \frac{d g}{d t}=-2 v_{d} k^{d-4} l_{1}^{d} \frac{3 x \lambda}{\kappa} \frac{1+\frac{x}{4}}{1+x}\left[s_{1}^{d}((2+x) \lambda \kappa)-s_{1}^{d}(-x \lambda \kappa)\right] \\
& \quad+2 v_{d} k^{d-4} l_{2}^{d} \lambda^{2}\left[9\left(1+\frac{x}{2}\right)^{2} s_{2}^{d}((2+x) \lambda \kappa)+\left(1-\frac{x}{2}\right)^{2} s_{2}^{d}(-x \lambda \kappa)\right] .
\end{aligned}
$$

Again the perturbative one-loop equations of $\lambda$ and $g$ are recovered for $d=4$ by putting $s_{2}^{4}=1$ in eqs. (17), (19) or eqs. (21), (23) and non-perturbative effects arise only through the mass dependence of the threshold functions.

With the three sets of evolution equations we are now prepared to study the phase transition in the two-scalar model in four dimensions. Fig. 1 shows the flow of the coupling $x$ and the dimensionless ratio $\kappa=\rho_{0} / k^{2}$ which indicates the position of the minimum of the potential. Trajectories are shown with decreasing scale $t=\ln (k / \Lambda)$, according to the sets of evolution equations given above with initial conditions set at some high momentum scale $\Lambda$. The phase diagram of fig. 1 is obtained for $\lambda(\Lambda)=0.1$. We observe the three phases S, AX and M separated by (solid) phase transition lines. The phase transition between the AX and M phase occurs always for $x=0$. This is easy to understand since $x=0$ corresponds to an enhanced $\mathrm{O}(2)$ symmetry. This symmetry is preserved if we start at $\Lambda$ with an $\mathrm{O}(2)$ symmetric potential. Since no trajectory can cross the line $x=0$ the phase diagram actually splits into two separate parts for $x>0$ and $x<0$. The phase 
transition between the AX and M phases as a function of $x(\Lambda)$ is of the second order and we observe that trajectories with small $x$ are attracted towards the transition line.

The phase transition between the S phase and SSB phase (AX or M phase) does not occur for $\kappa=0$ as in the tree approximation but rather for positive $\kappa(\Lambda)$. In the $\mathrm{S}$ phase near the phase transition a typical trajectory starts at $\Lambda$ near the critical line at $\kappa(\Lambda)>0$. It stays near the critical line for a certain range of scales, moving according to the arrows as dictated by the evolution equations in the $\mathrm{AX}$ or $\mathrm{M}$ phase. At a certain scale $k_{c}$ it deviates strongly from the critical line and $\kappa$ reaches zero at some scale $k_{s}>0$. Then the evolution equation of the symmetric regime has to be used, with boundary condition $m^{2}\left(k_{s}\right)=0$. The true phase diagram is, of course, three-dimensional and we only show a projection. The trajectories also have a component perpendicular to the projected plane. Since for small $\lambda$ the evolution of $\lambda$ is very slow, the projection on the plane with constant $\lambda$ gives a satisfactory picture.

On the critical line one observes three fixpoints: The "Ising fixpoint" at $x=-1$ corresponds to two disconnected scalar theories. The "Heisenberg fixpoint" at $x=0$ is characterized by an additional $\mathrm{O}(2)$ symmetry, and the "cubic fixpoint" is situated near $x=2$ (compare with eq. (12) ) The three points are partial fixpoints and become full fixpoints only in the limit where the running of $\lambda$ is neglected. (The only true full fixpoint is the Gaussian fixpoint for $\lambda=0$.) They become genuine fixpoints in three (or $4-\epsilon$ ) dimensions and we have used here their names common in statistical mechanics [18. The fixpoints at $x=-1$ and $x=2$ are repulsive and the $\mathrm{O}(2)$ symmetric one at $x=0$ is attractive.

In the region $-1<x<2$ the phase transition is of the second order. Near-critical trajectories stay very near the phase transition line before $\kappa$ deviates either towards zero (S phase) or infinity (SSB phase) at some scale $k_{c}$. Exactly on the phase transition the trajectories stay on the critical line for infinitely long "time", i.e. $k_{c} \rightarrow 0$. For $x<-1$ the critical trajectories run towards $x=-2$ where the quartic polynomial is unstable. We will show that in this region the phase transition is of the first order. The point $x=-1$ is a tricritical point separating the first and second order part of the phase diagram. Similarly, for $x>2$ the trajectories run to $x \rightarrow \infty(\lambda \rightarrow 0)$. This part of the phase diagram corresponds again to a first order transition with tricritical point at $x=2$. For the establishment of the first order character of the phase transition the two interesting regions are $x \simeq-2$ and $x \rightarrow \infty$. They lie on the left and right edges of the phase diagram in fig. 1.

In the regions $x \simeq-2$ and $x \rightarrow \infty(\lambda \simeq 0)$ the approximation of the effective average potential $U_{k}\left(\rho_{1}, \rho_{2}\right)$ by a quadratic polynomial in $\rho_{1}$ and $\rho_{2}$ becomes inadequate. Indeed, if one would insist on solving the corresponding evolution equations discussed before, one would find in these regions an asymptotic behaviour of the phase transition line characterized by fixpoints in $\kappa^{3} \lambda(g+\lambda)$ and $\kappa^{3} \lambda g$ respectively. They simulate a second order transition and are artefacts of an insufficient truncation. One has to account for

\footnotetext{
${ }^{4}$ The true fixpoint occurs exactly for $x=2$ and the small deviation from this value is due to a particularity of our truncation. For details see ref. [20].
} 
the possibility that the effective average potential has more than one local minima in these regions. In this case a description of the potential in terms of "local variables" at the absolute minimum, i.e. a polynomial expansion around this minimum, may be insufficient. A more global approach, which permits the simultaneous existence of more than one local minima, and monitors their properties as well as the differences in height between them, seems more appropriate.

In the remaining part we will extend the study by including higher derivative couplings as well as by monitoring the extrema of the potential simultaneously, in order to to get an accurate picture of the phase transition. We restrict ourselves to the region $x \rightarrow \infty$ $(\lambda \simeq 0)$ ๆ. We parametrize the effective average potential $U_{k}$ by eight parameters

$$
\begin{aligned}
U_{k} & =-\mu^{2}\left(\rho_{1}+\rho_{2}\right)+\frac{1}{2} \lambda_{0}\left(\rho_{1}^{2}+\rho_{2}^{2}\right)+g_{0} \rho_{1} \rho_{2} \\
& +\nu_{A}\left(\rho_{1}^{2} \rho_{2}+\rho_{1} \rho_{2}^{2}\right)+\nu_{B}\left(\rho_{1}^{3}+\rho_{2}^{3}\right) \\
& +\gamma_{A} \rho_{1}^{2} \rho_{2}^{2}+\gamma_{B}\left(\rho_{1}^{3} \rho_{2}+\rho_{1} \rho_{2}^{3}\right)+\gamma_{C}\left(\rho_{1}^{4}+\rho_{2}^{4}\right) .
\end{aligned}
$$

A convenient set of running parameters in this region is

$$
\begin{aligned}
& m_{0}^{2}=U_{1}(0)=U_{2}(0)=-\mu^{2} \quad \lambda_{0}=U_{11}(0)=U_{22}(0) \quad g_{0}=U_{12}(0) \\
& \rho_{0} \quad \text { from } \quad U_{1}\left(\rho_{0}\right)=0 \quad m^{2}=U_{2}\left(\rho_{0}\right) \\
& \lambda_{1}=U_{11}\left(\rho_{0}\right) \quad \lambda_{2}=U_{22}\left(\rho_{0}\right) \quad g=U_{12}\left(\rho_{0}\right) .
\end{aligned}
$$

Here we assume a vacuum expectation value at $\left(\rho_{10}=\rho_{0}, \rho_{20}=0\right)$ and the partial derivatives of the potential are evaluated at $\rho_{1}=0$ and $\rho_{1}=\rho_{0}$. The first three parameters describe the potential at the origin while the last five specify the minimum at $\rho_{1}=\rho_{0}$. The set of evolution equations is now given by

$$
\begin{array}{rlrl}
\frac{d m_{0}^{2}}{d t} & =\xi_{1}(0) & \frac{d \lambda_{0}}{d t}=\xi_{11}(0) & \frac{d g_{0}}{d t}=\xi_{12}(0) \\
\frac{d \rho_{0}}{d t} & =-\frac{\xi_{1}\left(\rho_{0}\right)}{U_{11}\left(\rho_{0}\right)} \quad \frac{d m^{2}}{d t}=\xi_{2}\left(\rho_{0}\right)+U_{12}\left(\rho_{0}\right) \frac{d \rho_{0}}{d t} \\
\frac{d \lambda_{1}}{d t} & =\xi_{11}\left(\rho_{0}\right)+U_{111}\left(\rho_{0}\right) \frac{d \rho_{0}}{d t} & \frac{d \lambda_{2}}{d t}=\xi_{22}\left(\rho_{0}\right)+U_{122}\left(\rho_{0}\right) \frac{d \rho_{0}}{d t} \\
\frac{d g}{d t}=\xi_{12}\left(\rho_{0}\right)+U_{112}\left(\rho_{0}\right) \frac{d \rho_{0}}{d t}, & &
\end{array}
$$

where $\xi=\partial U_{k} / \partial t\left(\rho_{1}, \rho_{2}\right)$ is defined in eq. (7), with subscripts again denoting partial derivation in the $\rho$-directions. The quantities on r.h.s. of the flow equations (26) are computed by neglecting terms with more than four derivatives with respect to $\rho_{1}, \rho_{2}$. The remaining partial derivatives (as for example $U_{1122}$ ) are expressed in terms of the parameters defined in eqs. (25) by use of the polynomial approximation of eq. (24). This leads to a system of eight coupled non-linear differential equations ?.

\footnotetext{
${ }^{5}$ The region $x \simeq-2$ can be mapped onto this region by a field transformation [20].

${ }^{6}$ The reduced system of three equations $(16),(17),(19)$ is obtained by the truncation $\nu_{A}=\nu_{B}=\gamma_{A}=$ $\gamma_{B}=\gamma_{C}=0$ and selecting the couplings $\rho_{0}, \lambda_{1}$ and $g$.
} 
We have solved this system numerically, starting with a classical potential given by eq. (11) and $\mu^{2}(\Lambda) / \Lambda^{2}=2.22 \times 10^{-4}, \lambda(\Lambda)=1 / 90, g(\Lambda)=1 / 10$. As long as $\lambda_{1}(k)$ remains larger than about $10^{-4}$ we see small quantitative but no qualitative differences as compared to the reduced system of eqs. (16), (17),(19). The trajectories look very similar to the ones of a second order phase transition. Only if we start with $\kappa(\Lambda)$ so close to the critical value that $\lambda_{1}(k)$ runs to values much smaller than $10^{-4}$ the first order character of the transition is revealed. We have depicted the phase diagram for small values of $\lambda_{1}$ in fig. 2, where we show the trajectories in the $\left(\lambda_{1}, \kappa\right)$ plane. The third relevant $\square$ coupling $g$ remains almost constant in this part of the phase diagram. When starting the trajectories in the lower right corner of fig. 2 all the remaining "irrelevant" parameters $\left(m_{0}^{2}, \lambda_{0}, g_{0}, m^{2}, \lambda_{2}\right)$ have to be set with very high precision equal to the values they take after the running along the (almost) critical trajectory for $\lambda_{1}(k)>10^{-4}$. Since this first part of the running extends over many orders of magnitude in the scale $k$ and the critical trajectory is unstable in the relevant $\kappa$-direction, care has to be taken to keep track of the critical trajectory accurately 8 .

The right and lower corner of the phase diagram of fig. 2 corresponds to an effective average potential $U_{k}$ with only one minimum. On the other hand, if a trajectory crosses the dashed line a second minimum develops and the upper left corner of fig. 2 corresponds to a potential with two local minima at $\rho=0$ and $\rho=\rho_{0} \neq 0$. To the left of the dasheddotted line the minimum at the origin is deeper than the minimum at $\rho_{0} \neq 0$. The curve $\mathrm{B}$ (which asymptotically approaches this line) corresponds to the phase transition line which separates the symmetric phase (all trajectories below B) from the phase with spontaneous symmetry breaking (all trajectories above B). The SSB phase can be subdivided into region $\mathrm{I}$ with only one minimum of $U_{k}$ and region II with minima at $\rho=0$ and $\rho=$ $\rho_{0} \neq 0$, the second one being deeper. The boundary trajectory A separates these regions. Similarly, in region III the potential has two local minima with the deeper one at zero. Region III is bounded by the spinodal curve C. This is the trajectory where $\lambda_{1}$ vanishes asymptotically for $k \rightarrow 0$. For trajectories below $\mathrm{C}$ and above $\mathrm{D}$ the minimum at $\rho_{0} \neq 0$ disappears for some scale $k_{2}>0$, where $\lambda_{1}\left(k_{2}\right)=0$. Finally, for trajectories below the boundary $\mathrm{D}$ the location of the minimum $\kappa(k)$ reaches zero for some scale $k_{s}>0$ with $\lambda_{1}\left(k_{2}\right)>0$. The behaviour of all trajectories above $\mathrm{A}$ and below $\mathrm{D}$ resembles closely a second order phase transition. The typical behaviour of the first order transition is only visible in a narrow range around the critical line in figs. 1 and 2 which corresponds to the region between the curves $\mathrm{A}$ and $\mathrm{D}$. We should mention at this point that for trajectories between $\mathrm{A}$ and $\mathrm{D}$ we have stopped the running of the couplings at some scale $k_{c n v}>0$ instead of computing the effective potential for $k \rightarrow 0$. The scale $k_{c n v}$ was chosen large

\footnotetext{
${ }^{7}$ We do not distinguish here between relevant and marginal couplings in the language of statistical mechanics.

8 The inherent numerical uncertainties do not permit to follow the almost critical trajectories in one sweep from $k=\Lambda$ to $k=0$. Algorithms with a "returning" of $\kappa$ at intermediate $k$ had to be developed. For the "starting point" for fig. 2 we used values for the parameters approximately given by $m_{0}^{2} / k^{2}=-1.36 \times 10^{-4}, \lambda_{0}=6.74 \times 10^{-5}, g_{0}=4.18 \times 10^{-2}, \kappa=\rho_{0} / k^{2}=2.09, m^{2} / k^{2}=8.73 \times 10^{-2}, \lambda_{1}=$ $6.39 \times 10^{-5}, \lambda_{2}=1.88 \times 10^{-5}, g=4.17 \times 10^{-2}$.
} 
enough so that $k^{2}+M_{1}^{2}>\frac{1}{4} k^{2}$, and $k^{2}+M_{2}^{2}>\frac{1}{4} k^{2}$ for all $\rho_{1}, \rho_{2}$ (cf. eqs. (7), (9)), and small enough so that the running couplings approached almost constant values. The potential with several minima therefore corresponds to the effective average potential $U_{k_{c n v}}$, rather than the effective potential $U_{0}$. In other words, the quantum fluctuations with momenta $q^{2}<k_{c n v}^{2}$ have not been included. The properties of the phase transition can actually be understood in terms of the shape of $U_{k_{c n v}}$. Including the quantum fluctuations with $q^{2}<k_{c n v}^{2}$ will lead to a convex effective potential $U_{0}$ [17. The physics related to the "approach to convexity" is not relevant for our purpose and rather obscures the simple picture presented above.

For $k>k_{c n v}$ we find that the contribution of the $\varphi_{1}$ modes to the running of $U_{11}\left(\rho_{1}, \rho_{2}=0, k\right)$ is very small as compared to the contribution of the $\varphi_{2}$ modes, once $\lambda_{1}(k)$ becomes smaller than $10^{-4}$. The $\varphi_{1}$ modes may therefore be neglected in this region If, corresponding to an omission of the first term on the r.h.s. of the flow equation (7). With

$$
M_{2}^{2}\left(\rho_{1}, \rho_{2}=0\right)=U_{2}\left(\rho_{1}\right)=m_{0}^{2}+g_{0} \rho_{1}+\frac{1}{2} \nu_{A} \rho_{1}^{2}+\frac{1}{6} \gamma_{B} \rho_{1}^{3}
$$

and neglecting the $k$-dependence of $m_{0}^{2}, g_{0}, \nu_{A}, \gamma_{B}$ we can easily integrate eq. (7). The solution for $k \rightarrow 0 \boxplus$ corresponds to a one-loop formula

$$
\begin{aligned}
U_{11}\left(\rho_{1}, 0\right)= & \lambda_{0}+\nu_{B} \rho_{1}+\frac{1}{2} \gamma_{C} \rho_{1}^{2} \\
& -\frac{1}{32 \pi^{2}}\left(g_{0}+\nu_{A} \rho_{1}+\frac{1}{2} \gamma_{B} \rho_{1}^{2}\right)^{2} \int_{0}^{\infty} d x x\left\{\frac{1}{\left(x+U_{2}\left(\rho_{1}\right)\right)^{2}}-\frac{1}{\left(P_{k_{1}}(x)+U_{2}\left(\rho_{1}\right)\right)^{2}}\right\} \\
& +\frac{1}{32 \pi^{2}}\left(\nu_{A}+\gamma_{B} \rho_{1}\right) \int_{0}^{\infty} d x x\left\{\frac{1}{x+U_{2}\left(\rho_{1}\right)}-\frac{1}{P_{k_{1}}(x)+U_{2}\left(\rho_{1}\right)}\right\} .
\end{aligned}
$$

Here $k_{1}$ may be chosen so that $\lambda_{1}\left(k_{1}\right)=10^{-4}$, with $P_{k_{1}}=x\left[1-\exp \left(-\frac{x}{k_{1}^{2}}\right)\right]^{-1}$. All couplings on the r.h.s. are taken at the scale $k_{1}$, with $\nu_{A}, \nu_{B}, \gamma_{B}$ and $\gamma_{C}$ re-expressed in terms of the couplings defined in eqs. (25). Omitting all couplings except $m_{0}^{2}, \lambda_{0}$ and $g_{0}$, we recognize the usual logarithmic behaviour of the Coleman-Weinberg potential

$$
U_{11}\left(\rho_{1}, 0\right) \approx \lambda_{0}-\frac{g_{0}^{2}}{32 \pi^{2}}\left[\ln \left(\frac{k_{1}^{2}+m_{0}^{2}+g_{0} \rho_{1}}{m_{0}^{2}+g_{0} \rho_{1}}\right)-\frac{k_{1}^{2}}{k_{1}^{2}+m_{0}^{2}+g_{0} \rho_{1}}\right] .
$$

Eq. (28) is in agreement with our numerical solution of the evolution equations and constitutes a quantitative improvement since no polynomial truncation of $U_{k}\left(\rho_{1}, 0\right)$ is imposed for $k<k_{1}$.

In conclusion, a combination of the flow equations for $\lambda_{1}(k)>10^{-4}$ with the "one-loop formula" (28) for $\lambda_{1}(k)<10^{-4}$ permits a reliable and quantitatively very precise understanding of the first order phase transition. Our non-perturbative method clearly answers

\footnotetext{
${ }^{9}$ Even though suspected by the smallness of $\lambda_{1}$ a similar statement cannot be proven within the standard perturbation theory since the saddle point appoximation breaks down for small $\rho_{1}$.

${ }^{10}$ Since only the fluctuations of $\varphi_{1}$ are related to the approach to convexity we can replace here $k_{c n v}$ with zero.
} 
the shortcomings of perturbation theory and firmly establishes the first order character of the transition for $\lambda(\Lambda)<\frac{1}{3} g(\Lambda)$. The situation is analogous to the abelian Higgs model for which a similar non-perturbative investigation has been performed recently [19]. Our method can be easily adapted to non-vanishing temperature or to the three-dimensional model relevant for statistical mechanics. In fact, we have presented our equations already for arbitrary dimensions $d$ and we emphasize that the usual perturbative infrared divergences in three dimensions are absent, due to the presence of an infrared cutoff $\sim k$. For a study of the temperature dependence of the effective potential it is sufficient to replace the threshold functions of eq. (14) by temperature dependent threshold functions [12], the modification arising from the discretization of the zero-component of the momentum. Combining the method presented here with the study of temperature dependence in ref. 12] constitutes an excellent basis for a quantitative understanding of the high temperature behaviour of the two-scalar model. This study is presented in detail in ref. 20.

\section{References}

[1] S. Coleman and E. Weinberg, Phys. Rev. D 7, 1888 (1973).

[2] A.D. Linde, JETP Lett. 23, 64 (1976); S. Weinberg, Phys. Rev. Lett. 36, 294 (1976).

[3] D.A. Kirzhnits and A. D. Linde, Ann. Phys. 101, 195 (1976); A. Guth and E. Weinberg, Phys. Rev. Lett. 45, 1131 (1980); E. Witten, Nucl. Phys. B 177, 477 (1981); A.I. Bochkarev and M.E. Shaposhnikov, Mod. Phys. Lett. A 2, 417 (1987); G. Anderson and L.J. Hall, Phys. Rev. D 45, 2685 (1992).

[4] V.A. Kuzmin, V.A. Rubakov, and M.E. Shaposhnikov, Phys. Lett. B 155, 36 (1985); M.E. Shaposhnikov, Nucl. Phys. B 287, 757 (1987); ibid 299, 797 (1988).

[5] See the Proceedings of the NATO Advanced Research Workshop: Electroweak physics and the early universe, Sintra, 1994 (Plenum Press) and references therein.

[6] A. Sakharov, JETP Letters 5, 24 (1967).

[7] ALEPH, DELPHI, L3, and OPAL collaborations, see M. Davier in the Proceedings of the International Lepton-Photon Symposium, Geneva, 1991, eds S. Hegarty, K. Potter, and E. Quercigh (World Scientific, Singapore, 1992).

[8] A. Bochkarev, and M.E. Shaposhnikov, Mod. Phys. Lett. A 2, 417 (1987).

[9] A.I. Bochkarev, S.V. Kuzmin and M.E. Shaposhnikov, Phys. Lett. B 244, 275 (1990); Phys. Rev. D 43, 369 (1991); N. Turok and J. Zadrozny, Nucl. Phys. B 369, 729 (1991); S. Myint, Phys. Lett. B 287, 325 (1992); J.R. Espinosa, M. Quiros and F. Zwirner, Phys. Lett. B 307, 106 (1993).

[10] A.H. Guth, Phys. Rev. D 23, 347 (1981).

[11] A.D. Linde, Phys. Lett. B 108, 389 (1982); A. Albrecht and P.J. Steinhardt, Phys. Rev. Lett. 48, 1220 (1982). 
[12] N. Tetradis and C. Wetterich, Nucl. Phys. B. 398, 659 (1993); preprint DESY-93-094 and HD-THEP-93-28, in press Nucl Phys. B; preprint DESY-93-128 and HD-THEP93-36, in press Int. J. Mod. Phys. A.

[13] C. Wetterich, Nucl. Phys. B 352, 529 (1991); Z. Phys. C 57, 451 (1993).

[14] L.P. Kadanoff, Physics 2, 263 (1966); K.G. Wilson, Phys. Rev. B 4 3174, 3184 (1971).

[15] C. Wetterich, Phys. Lett. B 301, 90 (1993).

[16] K.G. Wilson and I.G. Kogut, Phys. Rep. 12, 75 (1974); S. Weinberg, Critical phenomena for field theorists, in Erice Subnuc. Phys. 1 (1976); F. Wegner and A. Houghton, Phys. Rev. A 8, 401 (1978); J. Polchinski, Nucl. Phys. B 231, 269 (1984); A. Hasenfratz and P. Hasenfratz, Nucl. Phys. B 270, 685 (1986).

[17] A. Ringwald and C. Wetterich, Nucl. Phys. B 334, 506 (1990); N. Tetradis and C. Wetterich, Nucl. Phys. B 383, 197 (1992).

[18] D.J. Amit, Field theory, the renormalization group, and critical phenomena, World Scientific (1984).

[19] D. Litim, N. Tetradis and C. Wetterich, preprint OUTP-94-12 P and HD-THEP-9423.

[20] S. Bornholdt, N. Tetradis and C. Wetterich, preprint OUTP-94-13 P and HD-THEP94-28.

\section{Figures}

Fig. 1 Phase diagram for $\lambda(\Lambda)=0.1$.

Fig. 2 First order phase diagram in the region of small $\lambda_{1}$. 
This figure "fig1-1.png" is available in "png" format from: http://arxiv.org/ps/hep-th/9408132v1 
This figure "fig1-2.png" is available in "png" format from: http://arxiv.org/ps/hep-th/9408132v1 